\title{
Assessment of serum interleukin-35 as a diagnostic biomarker of neonatal early-onset sepsis
}

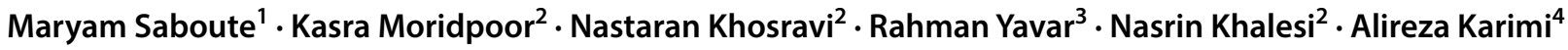

Received: 3 August 2019 / Accepted: 22 October 2019 / Published online: 12 November 2019

(c) Springer Nature Switzerland AG 2019

\begin{abstract}
Neonatal sepsis is a life-threatening disorder among infants, which is associated with high morbidity and mortality. In recent years, although the management of newborns has improved, early diagnosis and treatment of neonatal earlyonset sepsis (NEOS) are the major challenges for neonatologists in Neonatal intensive care units (NICUs). We assessed the diagnostic value of interleukins-35 (IL-35) in diagnosis of NEOS. A prospective process evaluation study was carried out in the NICU of Akbar-Abadi and Ali-Asghar Hospitals affiliated to Iran University of Medical Sciences from 2017 to 2018. Eighty hospitalized neonates with clinical suspicion of sepsis in the first $72 \mathrm{~h}$ of life entered the study by convenience or accidental sampling. Routine hematology tests regarding sepsis diagnosis were performed after admission. All subjects were assigned into two groups: newborns with proven sepsis and newborns with suspected sepsis. IL-35 levels in septic and unlikely infected hospitalized newborns were measured using Elisa Kit (ZellBio GmbH. Cat. No; ZB-10042C-H9648, Human Interleukin 35), and the results were compared. The mean IL-35 in serum samples from septic neonates was significantly higher than this variable in samples from unlikely infected newborns $(13.41 \pm 2.4 \mathrm{vs} .9 .02 \pm 2.6 \mathrm{pg} / \mathrm{ml} ; p<0.0001)$. The area under receiver-operating characteristic (ROC) curve for IL-35 was 0.895 ( $C l 95 \%=0.826-0.963 ; p=0.0001)$. The result of study showed serum IL-35 had a moderate accuracy (AUC $=0.895)$ for the diagnosis of NS. Therefore, serum IL-35 could be suggested as a predictive biomarker in neonatal early-onset sepsis. Further studies with larger sample size are strongly suggested.
\end{abstract}

Keywords Serum, IL-35 · Neonatal early-onset sepsis · Suspected sepsis · Predictive biomarker

\section{Introduction}

Neonatal early-onset sepsis (NEOS) defines as a systemic bacteremia with positive blood-cerebrospinal fluid culture, and life-threatening complications in the first $72 \mathrm{~h}$ of neonate's life [1]. Although the frequency of NEOS is relatively low (0.5-1.2 cases per 1000 live births), its morbidity and mortality rates are high [2-4]. Several maternal and neonatal causes are involved in the etiology of neonatal sepsis; colonization of organisms in the genitourinary tract, invasive procedures during pregnancy, prolonged rupture of membranes, instrumental delivery, prematurity, low birth weight, neonatal anomalies, and low Apgar scores are some of them. Group B Streptococcus, Escherichia coli, and Listeria monocytogenes are also reported as the most frequent pathogens in NEOS $[5,6]$.

Cytokines including interleukins (IL-2, IL-4, IL-6, IL-10, IL-12, IL-18, IL-23 ...), TNF- $a$, and IFN- $\gamma$ are the main members of body immune system $[7,8]$. Interleukin 35 (IL-35) as a heterodimer is a member of IL-12

\footnotetext{
$\triangle$ Nasrin Khalesi, nasrinkhalessi@yahoo.com; Maryam Saboute, saboutem@yahoo.com; Kasra Moridpoor, moridpoork@gmail.com; Nastaran Khosravi, nastarankhosravi@yahoo.com; Rahman Yavar, rahman.yavar@gmail.com; Alireza Karimi, karimi@kyudai.jp | ${ }^{1}$ Department of Pediatrics, Akbar-Abadi Hospital, Iran University of Medical Sciences, Tehran, Iran. ${ }^{2}$ Department of Pediatrics, Ali Asghar Hospital, Iran University of Medical Sciences, Tehran, Iran. ${ }^{3}$ Department of Genetics, Akbar-Abadi Hospital, Iran University of Medical Sciences, Tehran, Iran. ${ }^{4}$ Department of Mechanical Engineering, Kyushu University, 744 Motooka, Nishi-ku, Fukuoka 819-0395, Japan.
} 
family which is secreted by natural regulatory $T$ cells particularly cluster of differentiation $4\left(\mathrm{CD}^{+}\right)$and $\mathrm{T}$ helper (Th) cells and $\mathrm{CD} 4^{+} \mathrm{CD} 25^{+}$regulatory $\mathrm{T}$ (Treg) cells. IL-35 has potent anti-inflammatory and immunosuppressive properties in inflammation-related diseases $[9,10]$. The former studies have indicated significant alterations of serum IL-35 levels in patients with primary Sjögren's syndrome, cancerous diseases, chronic hepatitis, rheumatoid arthritis, encephalomyelitis, Hashimoto's thyroiditis, and respiratory-cardiovascular disorders [10-12]. Other studies have also indicated an association between plasma level of cytokines and sepsis; Du et al. [13] have shown a positive correlation between increase of IL-35 and sepsis severity in both adult and pediatric patients. Cao et al. have reported that serum levels of IL-35 in septic patients were significantly higher than this factor among their healthy counterparts. They also showed that the rise of serum concentration of IL-35 was consistent with the level of sepsis severity [14]. Machado et al. reported that proinflammatory and anti-inflammatory cytokines could be a probable biomarker for neonatal sepsis. They concluded that alterations of cytokine mediators could be a useful option for sepsis diagnosing or following up tool which may result early management and enhanced neonatal survival [15]. Sood et al. also demonstrated a significant increase in inflammatory cytokine mediators in neonatal sepsis in the first 3 weeks of life. These mediators were reported as the potential candidate biomarkers for early diagnosis of neonatal sepsis (before becoming positive the result of a blood culture sample) [16].

In recent years, although the management of newborns has improved, early diagnosis and treatment of NEOS are the major challenges for the neonatologist in Neonatal intensive care units (NICUs). In some septic cases, symptoms may be minimal, while in other cases, sepsis manifestations may be postponed for several days, resulting in wasting of golden time. In addition, diagnostic workups like blood and cerebrospinal fluid (CSF) cultures as well as other laboratory tests may need much time or may have not adequate sensitivity or specificity. On the other hand, antimicrobial multidrug-resistant organisms or unnecessary antibiotic treatment may be other complications for neonates clinically suspicious of septicemia [17]. It seems every novel diagnostic tool could be beneficial and of importance. However, very few data are available regarding the use of IL-35 in identifying neonatal sepsis, and therefore, we conducted this study prospective process evaluation, to assess the diagnostic value of IL-35 in diagnosis of neonatal early-onset sepsis in confirming or rejecting findings of previous studies.

\section{Methods and materials}

\subsection{Study design}

A prospective process evaluation study was carried out in the NICU of Akbar-Abadi and Ali-Asghar Hospitals affiliated to Iran University of Medical Sciences (Tehran-Iran) from 2017 to 2018. Based on an investigation by Du et al. in [13] and using formula as well as MedCalc software; 18.5 and area under the curve (AUC) 0.67 , the proposed sample size was considered 40 subjects for each group. Using formula, the proposed sample size of 80 , our study had a power of $80 \%$ and an alpha error of 0.05 .

$N=(Z 1-\alpha / 2) 2 p(1-p) / d 2$

$Z_{1}-\alpha / 2=1.96, p=0.67, d=0.75$

Eighty hospitalized neonates with clinical suspicion of sepsis in the first $72 \mathrm{~h}$ of life entered the study by convenience or accidental sampling. Patients with major congenital malformations, confirmed intrauterine viral infection, or lack of parental consent were excluded from the study.

Immediately after NICU admission, $4 \mathrm{CC}$ of venous blood was collected, centrifuged, stored in $-80^{\circ} \mathrm{C}$, labeled, and sent to the laboratory. Routine complete blood count $(C B C)$ was performed. C-reactive protein (CRP) by (QuikRead Go CRP system; sensitivity $\leq 1 \mathrm{mg} / \mathrm{ml}$ ), blood culture, and serum IL-35 by (Elisa Kit, ZellBio GmbH. Cat. No; ZB-10042C-H9648, Human Interleukin 35; Assay range $2.5-80 \mathrm{ng} / \mathrm{ml}$ (sensitivity; $0.1 \mathrm{ng} / \mathrm{ml}$ )) were measured. For all participants, lumbar puncture was also done, and samples were sent to the laboratory for CSF analysis. Detailed neonates' demographic and prenatal data gathered from medical records, as well as laboratory findings were recorded in some checklists.

Based on the laboratory results, perinatal history (positive history of chorioamnionitis, prolonged rupture of membrane or positive history of $\beta$ streptococci infection), and clinical manifestations, all subjects were assigned into two groups A and B.

Subjects in the group A were septic newborns with positive blood or CSF culture as well as subjects with negative cultures but with three clinical signs of sepsis. Sepsis signs were considered as below:

Temperature instability, mechanical ventilation requirement, heart rate dysrhythmia (Tachycardia; heart rate $>190$ and bradycardia; heart rate $<90$ ), poor feeding or feeding intolerance, abdominal distension, impaired peripheral blood circulation (pale skin, peripheral cyanosis, delayed capillary filling; more than $3 \mathrm{~s}$ ), neurological signs (lethargy, convulsion or irritability), abnormal laboratory findings including elevated CRP or abnormal hematologic cell count 
findings whole blood count $(\mathrm{WBC})<5 \times 109, \mathrm{CRP}>10 \mathrm{mg} / \mathrm{L}$, $\mathrm{I} / \mathrm{T}$ ratio $>0.12$ or platelet $\left.<10^{5} / \mu \mathrm{l}\right)[13]$.

Subjects in group B were unlikely infected newborns. Although they had negative blood and CSF cultures, one or two sepsis signs were diagnosed. Medical treatment in this group was stopped after $72 \mathrm{~h}$.

\subsection{Primary/secondary outcomes}

IL-35 levels in septic and unlikely infected hospitalized newborns were compared. Our primary objective was determining the predictive value of IL-35 in the diagnosis of neonatal early-onset sepsis among NICU-hospitalized sepsis-suspicious newborns. The secondary objectives were the assessment of the sepsis predictive ability of IL-35 for sepsis diagnosis.

\subsection{Ethical considerations}

The present study was taken from a medical student thesis with ID; IR.IUMS.REC1396.9511403001. Ethics approval was obtained from the Institutional Review Board of Iran University of Medical Sciences according to Helsinki Declaration. All participants' parents gave written consent before enrollment. All gathered data were considered confidential, and no extra cost was imposed on our participants.

\subsection{Statistical analysis}

Analyses were statistically performed by using software package SPSS version 19. We used Kolmogorov-Smirnov to examine normality of data related to two groups. Data with non-normal distributions were expressed as medians and interquartile ranges (IQR). Quantitative and qualitative variables with normal distributions were reported by mean and standard deviation (mean, SD) and number and percent $(n, \%)$, respectively. Student $t$ test and Mann-Whitney test were used for comparing quantitative data. Chi-square test and Fisher's exact test were used to compare qualitative variables such as sensitivity, specificity, positive predictive value (PPV), negative predictive value (NPV), and positive or negative likelihood ratios ( \pm LR) of IL-35 in predicting NEOS which were calculated. Receiver operative characteristics (ROC) curve was constructed for IL-35, and the areas under the ROC curve (AUC) were calculated. Moreover, the level of significance was considered as $p<0.05$.

\section{Results}

Eighty NICU-hospitalized neonates including 40 participants in each group $A$ and $B$ entered the study. The mean age of their mothers was $28.76 \pm 6.60$ years, and nine mothers (11.3\%) had a history of premature rupture of membrane. Mean gestational age, birth weight, head circumference, and length of neonates were $37.5 \pm 1.1$ weeks, 2834.2.6 $\pm 587.04 \mathrm{gr}, 33.6 \pm 2.4 \mathrm{~cm}, 48.3 \pm 3.4 \mathrm{~cm}$, respectively. A comparison of general parameters between the two septic and unlikely infected neonates is shown in Table 1 . No statistically significant differences were found in sex distribution, gestational age, birth weight, mode of delivery, Apgar scores at 1 and $5 \mathrm{~min}$, and number of gestations.

Regarding laboratory data, two subjects had positive blood or CSF cultures, while five cases had elevated CRP. The mean newborn's WBC counts on the first and third days were $(14.5 \pm 4.9) \times 10^{9}$ and $(10.8 \pm 5.7) \times 10^{9} / \mathrm{L}$. The mean platelet counts on the first and third days were also $(291.8 \pm 64.5) \times 10^{9}$ and $(283 \pm 84.1) \times 10^{9} / \mathrm{L}$. Immature-tototal neutrophils ratios (I/T ratio) for all participants were negative. WBC and PLT counts on the third day of hospital admission in septic neonates were also significantly higher than these variables in the other group $(p=0.03, p=0.03)$. On the one hand, no statistically significant differences

Table 1 Comparison of general parameters between the two septic and sepsis suspicious neonates

\begin{tabular}{|c|c|c|c|}
\hline Variable & $\begin{array}{l}\text { Septic neonates } \\
(n=40)\end{array}$ & $\begin{array}{l}\text { Sepsis-suspicious } \\
\text { neonates }(n=40)\end{array}$ & $P$ value \\
\hline \multicolumn{4}{|l|}{ Sex, $n(\%)$} \\
\hline Male & $29(72.5)$ & $31(77.5)$ & \multirow[t]{2}{*}{$N S^{*}$} \\
\hline Female & $11(27.5)$ & $9(22.5)$ & \\
\hline \multicolumn{4}{|c|}{ Gestational age, $w$} \\
\hline Min-max & $33-41$ & $34-40$ & \multirow[t]{2}{*}{$N S^{*}$} \\
\hline Mean \pm SD & $37.2 \pm 1.4$ & $37.8 \pm 1.7$ & \\
\hline \multicolumn{4}{|l|}{ Birth weight, gr } \\
\hline Min-max & $2080-3950$ & $2310-4050$ & \multirow[t]{2}{*}{$N S^{* *}$} \\
\hline Mean $\pm S D$ & $2810.2 \pm 587.04$ & $2858.3 \pm 674.1$ & \\
\hline \multicolumn{4}{|c|}{ Delivery mode, $n$ (\%) } \\
\hline NVD & $11(27.5)$ & $14(35)$ & \multirow[t]{2}{*}{$N S^{*}$} \\
\hline $\mathrm{C} / \mathrm{S}$ & $29(72.5)$ & $26(65)$ & \\
\hline \multicolumn{4}{|l|}{ Apgar 1 min, } \\
\hline Median (IQR) & $8.7(6-10)$ & $9.2(7-10)$ & $N S^{* * *}$ \\
\hline \multicolumn{4}{|l|}{ Apgar 5 min, } \\
\hline Median (IQR) & $9.5(9-10)$ & $9.8(8-10)$ & $N S^{* * *}$ \\
\hline \multicolumn{4}{|c|}{ Number of gestations, $n$ (\%) } \\
\hline Single & $37(92.5)$ & $38(95)$ & $N S^{* * * *}$ \\
\hline Twin & $3(7.5)$ & $2(5)$ & \\
\hline
\end{tabular}

$N$ number, $w$ week, gr gram, SD standard deviation, NVD normal vaginal delivery, $C / S$ cesarean section

*Chi-squared test

**Student's $t$ test

***Mann-Whitney test

****Fisher's exact test 
were observed between groups regarding CRP and CSF-blood culture results, and mean WBC-PLT counts on the first day of hospital admission $(p>0.05)$. Our results showed that the mean IL-35 in serum samples from septic neonates was significantly higher than this variable in unlikely infected neonates $(13.41 \pm 2.4$ vs. $9.02 \pm 2.6 \mathrm{pg} / \mathrm{ml}$; $p=0.0001$ ). Comparison of laboratory results in septic and sepsis suspicious neonates is shown in Table 2.

We also assessed the sepsis predictive ability of IL-35. ROC curves, calculating sensitivity, and specificity for IL-35 are shown in Fig. 1; based on ROC curves, AUC for IL-35 was 0.895 . This level was statistically significant as a neonatal early-onset sepsis diagnostic value $(\mathrm{Cl} 95 \%=0.826-0.963$; $p=0.0001)$. The cutoff values, specificity, sensitivity, and positive and negative predictive values (PPV \& NPV) of IL-35 in the diagnosis of neonatal early-onset sepsis are shown in Table 3. Our results have shown that cutoff $>8.05 \mathrm{pg} / \mathrm{ml}$ for IL-35 was the best diagnostic value for neonatal early-onset sepsis. Sensitivity and specificity with the cutoff $>8.05$ were 97 and $32 \%$.

\section{Discussion}

This study was done to evaluate the diagnosis or prediction of neonatal sepsis using IL-35. Neonatal sepsis because of high morbidity and mortality needs early and precise diagnosis [18]. Traditionally, the diagnosis of neonatal sepsis relies on determining the pathogen in the blood or CSF as well as the presence of clinical

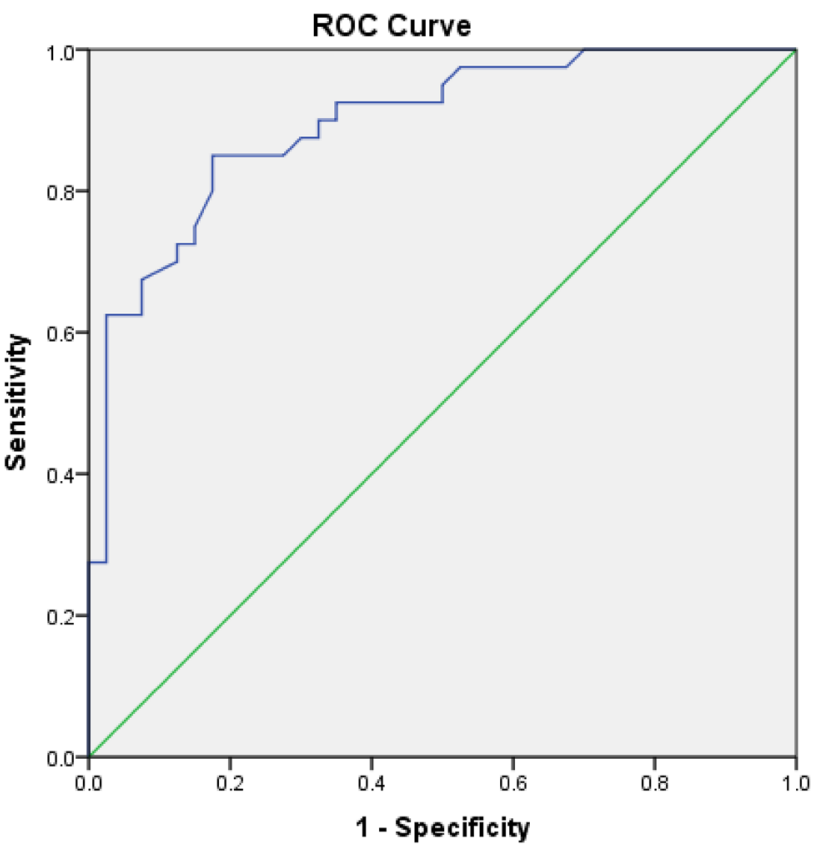

Diagonal segments are produced by ties.

Fig. 1 ROC of IL-35 level for diagnosis of early-onset sepsis among subjects AUC for IL-35; $0.895(\mathrm{Cl} 95 \%=0.826-0.963 ; p=0.0001$ )

signs and symptoms. On the other hand, some drawbacks related to these diagnostic tools were reported; several studies have pointed to low sensitivity, high false negativity, technical difficulties, and time consumption of blood culture test. Assessments of other laboratory
Table 2 Comparison of laboratory results in septic and sepsis suspicious neonates

\begin{tabular}{|c|c|c|c|}
\hline Variable & Septic neonates $(n=40)$ & $\begin{array}{l}\text { Sepsis suspicious } \\
\text { neonates }(n=40)\end{array}$ & $P$ value \\
\hline \multicolumn{4}{|l|}{ CSF culture, $n(\%)$} \\
\hline Positive & 0 & $1(2.5)$ & $0.314^{*}$ \\
\hline Negative & $40(100)$ & $39(97.5)$ & \\
\hline \multicolumn{4}{|l|}{ Blood culture, $n$ (\%) } \\
\hline Positive & 0 & $1(2.5)$ & $0.314^{*}$ \\
\hline Negative & $40(100)$ & $39(97.5)$ & \\
\hline \multicolumn{4}{|l|}{ CRP, $n(\%)$} \\
\hline Positive & $4(10)$ & $1(2.5)$ & $0.116^{*}$ \\
\hline Negative & $36(90)$ & $39(97.5)$ & \\
\hline WBC $\times 10^{9}$ on the first day, mean $\pm S D$ & $14.1 \pm 4.9$ & $14.96 \pm 5.01$ & $0.433^{* *}$ \\
\hline $\mathrm{PLT} \times 10^{9}$ on the first day, mean $\pm \mathrm{SD}$ & $294.15 \pm 65.83$ & $289.4 \pm 63.93$ & $0.744^{* *}$ \\
\hline $\mathrm{WBC} \times 10^{9}$ on the third day, mean $\pm S D$ & $11.84 \pm 5.63$ & $9.55 \pm 5.56$ & $0.03^{* *}$ \\
\hline $\mathrm{PLT} \times 10^{9}$ on the third day, mean $\pm \mathrm{SD}$ & $316.51 \pm 83.04$ & $244.51 \pm 70.70$ & $0.01^{* *}$ \\
\hline Serum IL-35, mean \pm SD & $13.41 \pm 2.4 \mathrm{pg} / \mathrm{ml}$ & $9.02 \pm 2.6 \mathrm{pg} / \mathrm{ml}$ & $0.0001^{* *}$ \\
\hline
\end{tabular}


Table 3 Sensitivity, specificity, positive predictive value, and negative predictive value of different cutoffs for diagnosis of early-onset sepsis among participants

\begin{tabular}{|c|c|c|c|c|c|}
\hline True positive & 39 & 37 & 34 & 32 & 29 \\
\hline False negative & 1 & 3 & 6 & 8 & 11 \\
\hline True negative & 13 & 20 & 29 & 33 & 34 \\
\hline False positive & 27 & 20 & 11 & 7 & 6 \\
\hline Sensitivity & $\begin{array}{l}97 \%(\mathrm{Cl} \\
\quad 95 \%=86-99 \%)\end{array}$ & $\begin{array}{l}92 \%(\mathrm{Cl} \\
95 \%=79-98 \%)\end{array}$ & $\begin{array}{l}85 \%(\mathrm{Cl} \\
\quad 95 \%=70-94 \%)\end{array}$ & $\begin{array}{l}80 \%(\mathrm{Cl} \\
\quad 95 \%=64-90 \%)\end{array}$ & $72 \%(\mathrm{Cl} 95 \%=56-85 \%)$ \\
\hline Specificity & $\begin{array}{l}32 \%(\mathrm{Cl} \\
\quad 95 \%=18-49 \%)\end{array}$ & $\begin{array}{l}50 \%(\mathrm{Cl} \\
\quad 95 \%=33-66 \%)\end{array}$ & $\begin{array}{l}72 \%(\mathrm{Cl} \\
95 \%=56-85 \%)\end{array}$ & $82 \%(\mathrm{Cl} 95=67-92 \%)$ & $85 \%(\mathrm{Cl} 95 \%=70-94 \%)$ \\
\hline $\begin{array}{l}\text { Positive predictive } \\
\text { value }\end{array}$ & $\begin{array}{l}59 \%(\mathrm{Cl} \\
\quad 95 \%=46-71 \%)\end{array}$ & $\begin{array}{l}64 \%(\mathrm{Cl} \\
95 \%=51-77 \%)\end{array}$ & $\begin{array}{l}75 \%(\mathrm{Cl} \\
\quad 95 \%=60-87 \%)\end{array}$ & $\begin{array}{l}82 \%(\mathrm{Cl} \\
\quad 95 \%=66-92 \%)\end{array}$ & $82 \%(\mathrm{Cl} 95 \%=66-93 \%)$ \\
\hline $\begin{array}{l}\text { Negative predictive } \\
\text { value }\end{array}$ & $\begin{array}{l}92 \% \\
\quad(\mathrm{Cl} 195 \%=66-99 \%)\end{array}$ & $\begin{array}{l}86 \%(\mathrm{Cl} \\
\quad 95 \%=66-97 \%)\end{array}$ & $\begin{array}{l}82 \%(\mathrm{Cl} \\
\quad 95 \%=66-93 \%)\end{array}$ & $\begin{array}{l}80 \%(\mathrm{Cl} \\
95 \%=65-91 \%)\end{array}$ & $75 \%(\mathrm{Cl} 95 \%=60-87 \%)$ \\
\hline Accuracy & $\begin{array}{l}65 \% \\
\quad(\mathrm{C} 195 \%=53-75 \%)\end{array}$ & $71(\mathrm{Cl} 95 \%=60-80 \%)$ & $\begin{array}{l}78 \%(\mathrm{Cl} \\
95 \%=68-87 \%)\end{array}$ & $\begin{array}{l}81 \%(\mathrm{Cl} \\
\quad 95 \%=70-89 \%)\end{array}$ & $78 \%(\mathrm{Cl} 95 \%=68-78 \%)$ \\
\hline $\begin{array}{l}\text { Positive likelihood } \\
\text { ratio }\end{array}$ & $1.44(1.15-1.80)$ & $1.85(1.34-2.55)$ & $3.09(1.83-5.19)$ & $4.57(2.19-9.11)$ & $4.83(2.25-10.35)$ \\
\hline $\begin{array}{l}\text { Negative likelihood } \\
\text { ratio }\end{array}$ & $13(1.78-94.73)$ & $6.66(2.15-20.67)$ & $4.83(2.25-10.35)$ & $4.12(2.18-7.79)$ & $3.09(1.83-5.19)$ \\
\hline Odds ratio & $18.77(2.31-152.15)$ & $12.33(3.26-46.62)$ & $14.93(4.91-45.38)$ & $18.85(6.12-58.07)$ & $14.93(4.91-45.38)$ \\
\hline
\end{tabular}

blood factors like C-reactive protein are also not specific enough to rely on them as the sepsis biomarkers. Clinical signs and symptoms are not specific, as well. Sepsis overestimation and overtreatment are also reported as the other challenging complications that should be extremely avoided [17, 19-21].

Recent investigations have introduced different sepsis diagnostic biomarkers to determine their utility for early detection of NEOS, leading better neonatal outcomes; IL-6, presepsin, serum interleukin-33, Interleukin-27, salivary C-reactive protein, mean platelet volume, and neutrophil/lymphocyte ratio are some of them [21-24].

In the present study, IL-35 levels in septic and neonates hospitalized newborns were compared to determine the predictive value of IL-35 as a biomarker in the diagnosis of NEOS. To our knowledge, there are very few investigations to assess this correlation [13, 14]. Our results demonstrated that the mean IL-35 in serum samples from septic neonates was significantly higher than this mediator in unlikely infected neonates $(13.41 \pm 2.4$, $9.02 \pm 2.6 ; p=0.0001)$. IL-35 is a proinflammatory and anti-inflammatory cytokine. It seems that immune cells including Treg, dendritic cells, macrophages, monocytes, and vascular endothelial and smooth muscle cells are responsible for increased serum IL-35 during sepsis [14]. In accordance with our results, Du et al. have indicated that of 157 enrolled subjects in the first $72 \mathrm{~h}$ of life; serum IL-35 concentration in sepsis infected neonates was significantly higher than this factor in unlikely infected subjects (median 36.4 vs. $27.1 \mathrm{pg} / \mathrm{ml}, p<0.001$ ) [13]. Cao et al. have also shown that serum IL-35 concentrations in 20 septic children were significantly higher than in 20 healthy counterparts $(p<0.01)$ [14].

Moreover, we suppose that serum IL-35 can be suggested as a predictive biomarker in NEOS. Based on ROC curve analysis, AUC for IL-35 was $0.895(\mathrm{Cl}$ $95 \%=0.826-0.963 ; p=0.0001$ ). Our results have also shown that the cutoff $>8.05 \mathrm{pg} / \mathrm{ml}$ for IL-35 with high negative predictive value and accuracy had a sensitivity of $97 \%$ and specificity of $32 \%$. So we suggested that, besides other laboratory tests and sepsis signs, assessment of serum IL-35 could be considered as a screening test in neonates with clinical suspicion of early-onset sepsis. This screening test could be easy, noninvasive, safe, and reasonable regarding the price. This finding is compatible with other studies; Du et al. pointed to IL-35 as a novel diagnostic biomarker with a high AUC (0.756) in NEOS. With cutoff $31.7 \mathrm{pg} / \mathrm{ml}$, the test had a sensitivity of $78.48 \%$ and specificity of $66.67 \%$ [13]. However, it is supposed that the difference in the levels of cutoff may be due to differences in the laboratory methods. Cao et al. have also indicated that serum IL-35 as a biomarker may provide some prognostic information in septic cases; the AUC for serum IL-35 level was 0.754 (CI 95\%, 0.590-0.918) [14].

According to our results, WBC and PLT counts on the third day of hospital admission in septic neonates were significantly higher than these variables in the other group $(p=0.03, p=0.03)$. On the other hand, Simonsen et al. [5] indicated a poor positive predictive value for WBC counts for sepsis diagnosis. In consistent with this finding, Hornik et al. have demonstrated that increased levels of both WBC and platelet counts after 3 days in neonates with negative 
culture were more notable than in neonates with positive culture. They showed significant correlations between the decrease in WBC-platelet counts on the third day with increase in odds of infection-related EOS [25].

Although the strengths of this study include the large sample size, availability of data related to hematologic tests, and showing the diagnostic value of serum IL-35 in ENOS, there are some limitations. We did not assess the correlation between serum IL-35 and the severity of sepsis. We also could not evaluate our cases regarding type of pathogens responsible for sepsis. Correlation between IL-35 concentrations and late-onset sepsis, as well as possibility of morbidity and mortality, was not assessed. Such investigations could provide some informative and beneficial data. One of other limitations in this study is inability to generalize the data. It would be important to enroll the healthy neonates to our study for comparing the results between the three groups septic, sepsis suspicious, and healthy neonates. Unfortunately, due to cost of laboratory test and kit and limitation of import of kits, it was not possible to perform. Further studies with larger sample size are strongly suggested.

\section{Conclusion}

The reference standard for the diagnosis of sepsis is a positive blood culture, which has various practical limitations. The early diagnosis of the NEOS certainly enhances therapeutic efforts leading to better outcomes. In this study, we identified serum IL-35 had a moderate accuracy (AUC $=0.895$ ) for the diagnosis of NS. This is one of the first studies to explore the role of IL-35 in predicting neonatal sepsis. Based on our results, serum IL-35 could be suggested as a predictive biomarker in neonatal early-onset sepsis. Because of the prospective design of the study and the clear definition of clinical and laboratory criteria used to diagnose NEOS, the results could be considered reliable. However, it is important to conduct adequately powered prospective multicenter studies to continue to establish the accuracy and safety of utilizing such biomarkers of neonatal sepsis.

Acknowledgements This study was supported by Iran University of medical sciences (IUMS). We also acknowledge the parents for their participation in this study. Also, the authors would like to thank Dr. Leila Allahqoli, Iran University of Medical Sciences, Tehran, Iran, for helping her in revising the paper.

\section{Compliance with ethical standards}

Conflict of interest The authors declare that they have no conflict of interest.
Human and animals rights Ethics approval was obtained from the institutional review board of Iran University of Medical Sciences according to Helsinki Declaration. Parents of all participants gave written consent before enrollment.

Informed consent Informed consent was obtained.

\section{References}

1. Sherrianne N, Strunk T, Jiang P, Muk T, Sangild P, Currie A (2018) Precision medicine for neonatal sepsis. Front Mol Biosci 5:70. https://doi.org/10.3389/fmolb.2018.00070

2. Fell DB, Hawken S, Wong CA, Lindsay AW, Malia SQ, Pranesh C et al (2017) Using newborn screening analytes to identify cases of neonatal sepsis. Sci Rep 7(1):18020. https://doi.org/10.1038/ s41598-017-18371-1

3. Puopolo KM, Escobar GJ (2013) Early-onset sepsis: a predictive model based on maternal risk factors. Curr Opin Pediatr 25(2):161-166. https://doi.org/10.1097/mop.0b013e32835e1f96

4. Mukhopadhyay S, Puopolo KM (2012) Risk assessment in neonatal early onset sepsis. Semin Perinatol 36(6):408-415. https:// doi.org/10.1053/j.semperi.2012.06.002

5. Simonsen KA, Anderson-Berry AL, Delair SF et al (2014) Earlyonset neonatal sepsis. Clin Microbiol Rev 27(1):21-47

6. Polin RA (2012) Management of neonates with suspected or proven early-onset bacterial sepsis. Pediatrics 129(5):1006-1015

7. Hodge G, Haslam R, McPhee A, Sepulveda H, Morgan E, Nicholson I et al (2004) Rapid simultaneous measurement of multiple cytokines using $100 \mu \mathrm{l} \mathrm{sample} \mathrm{volumes-association} \mathrm{with} \mathrm{neo-}$ natal sepsis. Clin Exp Immunol 137(2):402-407. https://doi.org /10.1111/j.1365-2249.2004.02529.x

8. Khosravi N, Khalesi N, Noorbakhsh S, Javadinia S, Asgarian R, Tabatabai A (2014) The relationship between cerebrospinal fluid C-reactive protein and neonatal meningitis. Tehran Univ Med J 71(11):723-728

9. Xiaoning $Z$, Zhiqiang $Z$, Zhiqiang $\mathrm{H}$, Mingyan J, Li J, Jinghua $Y$ et al (2018) Interleukin 35 induced Th2 and Tregs bias under normal conditions in mice. Peer J 6:e5638. https://doi.org/10.7717/ peerj.5638

10. Meiling $H$, Yuxuan $L$, Siyan $L$, Shenyi J, Lin $Y$, Liping $X$, Hui $S$, Jing $L$ (2018) Elevation of serum IL-35 in patients with primary Sjögren's syndrome. J Interferon Cytokine Res. https://doi. org/10.1089/jir.2018.0059

11. Yanhui $M$, Lei $C$, Guohua $X$, Yunlan $Z$, Chaoyan $Y$, Xiangliang $Y$ et al (2016) Elevated level of Interleukin-35 in colorectal cancer induces conversion of T cells into iTr35 by activating STAT1/ STAT3. Oncotarget 7(45):73003-73015. https://doi.org/10.18632 /oncotarget.12193

12. Bello RO, Chin V, Isnadi M, Abd Majid R, Abdullah M, Lee T, Zakaria $Z$ et al (2018) The role, involvement and function(s) of Interleukin-35 and Interleukin-37 in disease pathogenesis. Int J Mol Sci 19(4):1149. https://doi.org/10.3390/ijms19041149

13. Du WX, He Y, Jiang HY, Ai Q, Yu JL (2016) Interleukin 35: a novel candidate biomarker to diagnose early onset sepsis in neonates. Clin Chim Acta 462:90-95. https://doi.org/10.1016/j. cca.2016.09.005

14. Cao J, Xu F, Lin S, Tao X, Xiang Y, Lai X et al (2015) IL-35 is elevated in clinical and experimental sepsis and mediates inflammation. Clin Immunol 161(2):89-95. https://doi.org/10.1016/j. clim.2015.08.016

15. Machado R, Danilo F, Marcos V, Liliana B, Renata M, Maria L et al (2014) Neonatal sepsis and inflammatory mediators. Mediators Inflamm 2014:269681. https://doi.org/10.1155/2014/269681 
16. Sood B, Seetha S, Schelonka R, Shampa S, Danny K, Sánchez $P$ et al (2012) Cytokine profiles of preterm neonates with fungal and bacterial sepsis. Pediatr Res 72(2):212-220. https://doi. org/10.1038/pr.2012.56

17. Akila P, Prashant V, Praveen K, Prashanth S, Vijaykumar G et al (2013) Comparative assessment of cytokines and other inflammatory markers for the early diagnosis of neonatal sepsis-a case control study. PLoS One 8(7):e68426. https://doi. org/10.1371/journal.pone.0068426

18. Li Y, Ke J, Peng C, Wu F, Song Y (2018) microRNA-300/NAMPT regulates inflammatory responses through activation of AMPK/ mTOR signaling pathway in neonatal sepsis. Biomed Pharmacother 108:271-279. https://doi.org/10.1016/j.biopha.2018.08.064

19. Halek J, Novak M, Medkova A, Furst T, Juranova J (2018) The role of nCD64 in the diagnosis of neonatal sepsis in preterm newborns. Biomed Pap Med Fac Univ Palacky Olomouc Czech Repub 162(4):284-288. https://doi.org/10.5507/bp.2018.033

20. Shaaban HA, Safwat N (2018) Mean platelet volume in preterm: a predictor of early onset neonatal sepsis. J Matern Fetal Neonatal Med. https://doi.org/10.1080/14767058.2018.1488161

21. Rashwan NI, Hassan MH, Mohey El-Deen ZM, Ahmed AE (2019) Validity of biomarkers in screening for neonatal sepsis - a single center -hospital based study. Pediatr Neonatol 60(2):149-155. https://doi.org/10.1016/j.pedneo.2018.05.001
22. Halil H, Tayman C, Buyuktiryaki M, Okur N, Cakir U, Serkant U (2018) Serum interleukin-33 as a biomarker in predicting neonatal sepsis in very low birth weight infants. Comb Chem High Throughput Screen. https://doi.org/10.2174/138620732166618 0911090656

23. Omran A, Maaroof A, Saleh MH, Abdelwahab A (2018) Salivary C-reactive protein, mean platelet volume and neutrophil lymphocyte ratio as diagnostic markers for neonatal sepsis. J Pediatr (Rio J) 94(1):82-87. https://doi.org/10.1016/j.jped.2017.03.006

24. He Y, Du WX, Jiang HY, Ai Q, Feng J, Liu Z, Yu JL (2017) Multiplex cytokine profiling identifies Interleukin-27 as a novel biomarker for neonatal early onset sepsis. Shock 47(2):140-147. https://doi. org/10.1097/shk.0000000000000753

25. Hornik CP, Benjamin DK, Becker KC, Benjamin DK, Li J, Clark RH et al (2012) Use of the complete blood cell count in early-onset neonatal sepsis. Pediatr Infect Dis J 31(8):799-802. https://doi. org/10.1097/inf.0b013e318256905c

Publisher's Note Springer Nature remains neutral with regard to jurisdictional claims in published maps and institutional affiliations. 